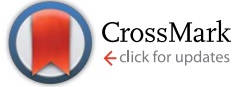

Cite this: RSC Adv., 2015, 5, 95149

Received 20th July 2015

Accepted 29th October 2015

DOI: $10.1039 / c 5 r a 14310 a$

www.rsc.org/advances

\section{Dispersion analysis of carbon nanotubes, carbon onions, and nanodiamonds for their application as reinforcement phase in nickel metal matrix composites}

\begin{abstract}
L. Reinert, ${ }^{\star a}$ M. Zeiger, ${ }^{\text {ab }}$ S. Suárez, ${ }^{a}$ V. Presser ${ }^{a b}$ and F. Mücklich ${ }^{a}$
Dispersions of multi-wall carbon nanotubes, onion-like carbon, and nanodiamonds in ethylene glycol are produced using a homogenizer and an ultrasonic bath, altering the treatment time. The dispersed particles are then used as reinforcement phase for nickel matrix composites. These nanoparticles are chosen to represent different carbon hybridization states $\left(\mathrm{sp}^{2}\right.$ vs. $\mathrm{sp}^{3}$ ) or a different particle geometry (OD vs. 1D). This allows for a systematic investigation of the effect of named differences on the dispersibility in the solvent and in the composite, as well as the mechanical reinforcement effect. A comprehensive suite of complementary analytical methods are employed, including transmission electron microscopy, Raman spectroscopy, dynamic light scattering, sedimentation analysis, zeta-potential measurements, scanning electron microscopy, electron back scatter diffraction, and Vickers microhardness measurements. It can be concluded that the maximum achievable dispersion grade in the solvent is similar, not altering the structural integrity of the particles. However, nanodiamonds show the best dispersion stability, followed by onion-like carbon, and finally multi-walled carbon nanotubes. The distribution and agglomerate sizes of the particles within the composites are in good agreement with the dispersion analysis, which is finally correlated with a maximum grain refinement by a factor of 3 and a maximum mechanical reinforcement effect for nanodiamonds.
\end{abstract}

\section{Introduction}

Tailoring of the physical properties of composite materials has been an extensively studied topic over the last decades. Lately, carbon nanomaterials have been introduced as very promising reinforcement candidates in composites to increase strength, ${ }^{\mathbf{1 - 3}}$ hardness, ${ }^{4-6}$ electrical and thermal conductivity, ${ }^{5,7,8}$ thermomechanical stability, ${ }^{9}$ and reduce friction and wear. ${ }^{10-12}$ Carbon allotropes can be found in a wide range of morphologies and configurations; they are also attractive reinforcement phases mainly due to their outstanding properties and low density compared to other reinforcement materials. ${ }^{13-15}$

For our work, we focus on three types of carbon nanoparticles (CNPs), namely multi-wall carbon nanotubes (MWCNT) ${ }^{16}$ nanodiamonds (ND) ${ }^{15}$ and onion-like carbons (OLC). ${ }^{17}$ CNTs can be described as helical hollow cylinders of graphitic carbon $\left(\mathrm{sp}^{2}\right.$ hybridized) having one or multiple shells and a high aspect ratio (i.e., quasi one-dimensional). ${ }^{16} \mathrm{ND}$ are spherical diamond nanoparticles with a diamond lattice spacing of $0.21 \mathrm{~nm}$ and a primary

${ }^{a}$ Department of Materials Science and Engineering, Saarland University, 66123 Saarbrücken, Germany. E-mail: l.reinert@mx.uni-saarland.de

${ }^{b} I N M-L e i b n i z$ Institute for New Materials, Saarland University, 66123 Saarbrücken, Germany particle diameter of around $5 \mathrm{~nm}$ (i.e., quasi zero dimensional). ${ }^{15}$ Due to the synthesis via detonation reaction, ND are covered with functional groups and amorphous carbon layers. ND particles also can form agglomerates with sizes up to hundreds of nanometers. ${ }^{18}$ Thermal annealing in inert atmosphere or vacuum allows to transform ND to OLC. ${ }^{19}$ The latter are also known as carbon onions and can be understood as multi-shell fullerene nanoparticles (i.e., quasi zero dimensional) with a typical spacing of 0.34-0.36 nm between the outer shells. ${ }^{20,21}$ Depending on the synthesis method and conditions, the ratio between $\mathrm{sp}^{3}$ - and $\mathrm{sp}^{2}$ hybridized carbon can be tuned, as well as the degree of carbon ordering. ${ }^{19,22,23}$

We chose these three particular CNPs as they show either a different carbon hybridization state or a different particle geometry. This allows for a systematic investigation of the effect of those differences on the particle distribution and the properties in a composite material.

Since a fine and homogeneous distribution of the particles is required to achieve an optimum reinforcing effect, ${ }^{8}$ the main drawback of working with CNPs is their notorious tendency to agglomerate due to intermolecular forces like dipole-dipole forces or van-der-Waals forces. ${ }^{24-26}$ This behavior is highly dependent on the synthesis conditions. ${ }^{\mathbf{1 8 , 1 9 , 2 2 , 2 3}}$ CNP agglomerates tend to reduce the intrinsic properties of the CNP and also 
the matrix-reinforcement interface. ${ }^{8}$ Hence, finding a way to efficiently disaggregate and distribute CNPs within a matrix material without significantly altering their structure or properties is the key to fully exploit the potential of CNP composites.

There are two mayor strategies for facile CNP de-agglomeration: mechanical milling and dispersion in solvents. Focusing on $\mathrm{sp}^{2}$ hybridized CNPs, mechanical milling (e.g., ball milling) is known to unbundle agglomerates efficiently, but this process also affects the particle morphology and defect state, in particular for CNT and OLC. ${ }^{27-29}$ The second possibility can be sub-classified into two alternatives: (1) the nanoparticles can be functionalized (covalently or non-covalently), or (2) be dispersed in a suitable solvent without prior functionalization. ${ }^{30}$ The covalent functionalization implies the dissociation of a C-C bond or the utilization of dangling bonds to enable surface functionalization (e.g., hydroxyl, carboxyl or carbonyl groups), rendering the particles dispersible. This treatment procedure involves the opening of the structure, generating a weak point where the particle might mechanically fail. Furthermore, it does not only affect the mechanical properties of the particle, but many physical properties of the CNPs are diminished as well. ${ }^{31-33}$

All of the CNPs in this study show a strong tendency to agglomerate in their native (as received) form. In case of CNTs, their high aspect ratio, high flexibility, and strong attractive forces lead to a strong tendency to form bundles. ${ }^{24,25}$ During unbundling, to avoid a change of the graphitic structure, it is possible to functionalize the CNT non-covalently by physisorption of molecules. This implies the use of surfactants, polymers, or organic molecule wrapping. ${ }^{34-37}$ In several studies, the optimum CNT/solvent ratio for different solvents has been studied and some solvent systems have been identified that do not require prior carbon functionalization. ${ }^{30}$

Detonation nanodiamonds also show a strong tendency to form agglomerates. ${ }^{26}$ During the synthesis, the detonation wave during the synthesis may generate dangling bonds at the surface of the ND that react and form covalent interparticle bonds, graphitic shells or surface functional groups which interact via dipole-dipole or van-der-Waals forces leading to agglomerates. ${ }^{26}$ Similar to CNT, one way to disperse NDs is by covalent functionalization; depending on the target solvent, one would adapt either hydrophobic or hydrophilic functional groups on the surface of ND particles. ${ }^{38,39}$ This can for example be achieved by using organic molecules like polymer brushes of polystyrene and poly $(t \text {-butyl methacrylate })^{\mathbf{4 0}}$ or by an esterification reaction of carboxylic acid chlorides on hydroxylated nanodiamonds. ${ }^{\mathbf{4 1}}$ Also a non-covalent functionalization of ND is possible, using different kinds of surfactants combined with prior chemical or thermal oxidation. ${ }^{39,42,43}$

OLCs derived by thermal annealing of ND also tend to form agglomerates; depending on the applied synthesis conditions, agglomeration is a result of onsetting particle-particle sintering and strong intermolecular forces. ${ }^{19,22,23}$ Covalent functionalization may strongly improve the solubility of carbon onions in suitable solvents; for example, Liu et al. successfully used fluorination to severely improve the solubility in organic solvents. ${ }^{44}$ It is also possible to use a surfactant like polyacrylic acid to significantly reduce agglomeration of OLCs. ${ }^{29}$
Our work was motivated by the need to find improved ways to modify metal matrix composites by employing suitable CNP materials. We exemplify the potentials and limitations of CNT, OLC, or ND nanoparticles for nickel matrix composites. As a first step, we employed a homogenizer and ultrasonication to derive dispersions of CNT, OLC, or ND in ethylene glycol. We excluded chemical post-synthesis treatment since the latter prohibits a proper analysis of the influence of the hybridization state of carbon atoms and the particle geometry on the particles dispersion. Such post-synthesis treatments would also add an additional step to the process while our goal was to explore the facile production of composite materials. To the best of our knowledge, this is the first systematic comparison of the dispersion of CNTs, OLCs, or NDs in a metal matrix. Dynamic light scattering (DLS) was used to determine the particle agglomeration sizes in the solvent. Additionally, the stability of the dispersions was investigated by sedimentation analysis and zeta-potential measurements. The structural integrity of the particles was analyzed by Raman spectroscopy as a function of the processing time by the homogenizer or the ultrasonic bath.

After this step, we adapted the CNPs to a sintered nickel matrix composite. We correlated the results from the comprehensive particle characterization with the distribution of the particles within the nickel matrix, the obtained microstructure, and the resulting mechanical reinforcement effect analyzed by scanning electron microscopy (SEM), electron back scatter diffraction (EBSD), and Vickers microhardness measurements. Nickel was chosen as matrix material as it forms only metastable carbides under very specific conditions, therefore being suited for a reinforcement based on CNPs with the parasitic emergence of interphases. ${ }^{45}$ Additionally, the distribution of reinforcing particles in a metal matrix composite as well as their properties strongly influences the development of the reinforcing effect. ${ }^{46}$ Therefore, for their subsequent utilization as precursors in composite materials manufacturing, it is of utmost importance to understand the disaggregation and colloidal stability of different (nonfunctionalized) CNPs in solvents.

\section{Experimental}

\subsection{Processing}

In this work, dispersions of MWCNT (Baytubes C150P from Bayer, purity $>95 \%$, individual particle diameter of 5-20 nm), ND (NaBond Technologies Co., purity $>98 \%$, individual particle diameter 4-8 $\mathrm{nm}$ ), and nanodiamond-derived $\mathrm{OLC}^{19}$ in ethylene glycol were analyzed. OLC were synthesized from the same ND powder mentioned before by annealing the material in graphite crucibles (30 $\mathrm{mm}$ in diameter and $20 \mathrm{~mm}$ in height) in a vacuum furnace with tungsten heaters (model: 1100-3580-W1, Thermal Technology Inc.) using a heating and cooling rate of 15 ${ }^{\circ} \mathrm{C} \min ^{-1}$. The chamber pressure is between $10 \mathrm{mPa}$ and 100 $\mathrm{mPa}$. OLC are synthesized at $1750{ }^{\circ} \mathrm{C}$ with a holding time of $3 \mathrm{~h}$.

For the dispersion process, we used a homogenizer (WiseTis, Witeg) and an ultrasonic bath (Sonorex Super RK $514 \mathrm{BH}$ by Bandelin, $860 \mathrm{~W}, 35 \mathrm{kHz}$ ). In case of ultrasonic treatment, the strong shear force that can exfoliate the agglomerates comes from cavitation, which leads to a process of bubble formation, 
growth, and collapse. The efficiency of this process can be correlated to the solvent parameters like viscosity, vapor pressure and surface tension, as well as the time, sonication frequency, and intensity. ${ }^{30}$ Some of the most promising solvents for stable dispersions, like dimethylformamide (DMF), are highly toxic, which is complicating cost effective large-scale applications. $^{30}$ The solvent ethylene glycol has already been used successfully in other works and is not toxic. ${ }^{47}$ Furthermore, polyole like ethylene glycol allow for a subsequent functionalization of carbon nanoparticles or the deposition of metal particles onto the carbon nanoparticles, establishing the possibility of further research work on this topic. ${ }^{47}$ Hence, ethylene glycol is a versatile solvent and was therefore chosen for the present work. The concentration was kept constant for all three different CNP materials at 0.006 vol\%. This is calculated after having measured the densities by gas pycnometry with a Micromeritics AccuPyc 1330 gas pycnometer using helium (purity grade: 5.0). Before, the powders were dried in a furnace at $120{ }^{\circ} \mathrm{C}$ for $1 \mathrm{~h}$ to remove any amount of adsorbed water. After ten purges, each sample was analyzed by 20 separate measurements with a fill pressure of $134 \mathrm{~Pa}$ and an equilibration rate of $34 \mathrm{~Pa} \mathrm{~min}^{-1}$. The measured skeletal densities were $1.92 \pm 0.01 \mathrm{~g} \mathrm{~cm}^{-3}$ for CNT, $1.97 \pm 0.03 \mathrm{~g} \mathrm{~cm}^{-3}$ for OLC, and $3.30 \pm 0.01 \mathrm{~g} \mathrm{~cm}^{-3}$ for ND.

The obtained dispersions were used to manufacture nickel matrix composites with Ni dendritic powder (Alfa Aesar, -325 mesh) with a particle volume content of $6.5 \%$. This volume content is equivalent to 1.48 mass \% of CNT, 1.52 mass\% of OLC and 2.51 mass $\%$ of ND. The importance of the utilization of the volume fraction lies in the fact that it is the most direct way to compare the behavior to other composites (other matrices). In order to allow for a proper comparison of different carbon nanoparticles in the same metal matrix system, the volume amount of particles in the system must be kept constant instead of the mass fraction. A maximum reasonable concentration of CNTs in the nickel matrix was found to be 3 mass $\%$, which is equivalent to about 12.5 vol\%. ${ }^{45}$ With this concentration, a density of $98 \%$ could be reached and a the grain refinement effect was maximized using nickel matrix composites. Higher concentrations lead to a decrease of the reinforcement effect due to a more pronounced re-agglomeration of the CNTs. ${ }^{45}$ Based on this fact, a medium amount of the reinforcing carbon nanoparticles was chosen in this study.

After evaporating the solvent of the dispersions in a furnace at $150{ }^{\circ} \mathrm{C}$, the powder was pressed to green pellets (pressure of $990 \mathrm{MPa}$ ) which can be sintered in a hot uniaxial press (axial pressure of $264 \mathrm{MPa})$ in vacuum $\left(2 \times 10^{-6} \mathrm{mbar}\right)$ at $750{ }^{\circ} \mathrm{C}$ for $2.5 \mathrm{~h}$. More information on the synthesis process is provided elsewhere. ${ }^{48}$

\subsection{Characterization}

Samples were analyzed as a powder dispersed on lacey carbon grids using transmission electron microscopy (TEM) (JEOL $2100 \mathrm{~F}$ ) operated at $200 \mathrm{kV}$.

To characterize the dispersion in ethylene glycol, the hydrodynamic radius of the particle agglomerates in the solvent is measured using dynamic light scattering (ALV Compact Goniometer) with a wavelength of $532 \mathrm{~nm}$ as a function of the time spent in the homogenizer or the ultrasonic bath. All spectra were recorded directly after the dispersion process to represent the dispersion state at this time. These results were then correlated to the sedimentation characteristics of the particles, measured using a Lumisizer (LUM GmbH) with 200$4000 \mathrm{rpm}$ and a wavelength of $470 \mathrm{~nm}$, taking $\sim 24 \mathrm{~h}$ until all particles had settled. The dispersion stability was investigated by zeta-potential measurements with a particle charge detector (Mütek PCD-03 pH, BTG). Five dispersions of each type with the same particle volume concentration and a treatment of five minutes in the homogenizer and $20 \mathrm{~min}$ in the ultrasonic bath were measured and the resulting data was averaged. A conducting salt (ammonium acetate, purity $>97 \%$, Alfa Aesar) with a concentration of $10^{-3} \mathrm{~mol} \mathrm{dm}{ }^{-3}$ was added to increase the conductivity of the solvent ethylene glycol while keeping the $\mathrm{pH}$ constant at 7.6 thus allowing for the measurement. ${ }^{49}$

Raman spectra were recorded using an inVia Raman microscope (Renishaw) with an excitation wavelength of $532 \mathrm{~nm}$ $(2.33 \mathrm{eV})$ for the examination of CNT and OLC, as this wavelength is more sensitive to coupled modes induced by $\mathrm{sp}^{2}$ hybridization of carbon atoms. ${ }^{23}$ The data was recorded using a grating with 2400 lines per $\mathrm{mm}$, a $50 \times$-objective (numeric aperture: 0.9), a spectral resolution of $1.2 \mathrm{~cm}^{-1}$, and a laser power of $0.2 \mathrm{~mW}$. For ND, an UV-Raman spectrometer (developed by the Institute Jean-Lamour in Nancy, France) with a $50 \times-$ objective (numeric aperture: 0.55), a grating of 1800 lines per $\mathrm{mm}$, a spectral resolution of $1.2 \mathrm{~cm}^{-1}$, an excitation wavelength of $325 \mathrm{~nm}(3.81 \mathrm{eV})$, and a laser power of $0.2 \mathrm{~mW}$ was used due to the higher sensitivity to $\mathrm{sp}^{3}$-hybridization of the carbon atoms. ${ }^{23}$ The reference spectrum is measured using the asreceived agglomerated carbon nanoparticle powders. During the dispersion of the different carbon nanoparticles in the ultrasonic bath, an aliquot was taken out of the dispersions with a pipette at the particular times $(10,20$, and $120 \mathrm{~min})$ and deposited onto a silicon wafer. The solvent was subsequently evaporated at $150{ }^{\circ} \mathrm{C}$ in a furnace. Finally, the agglomerates on the silicon wafer were measured with Raman spectroscopy to obtain the provided spectra. All Raman spectra were recorded three times with an acquisition time of $10 \mathrm{~s}$ to eliminate cosmic rays and to improve the signal-to-noise ratio. Additionally, a linear baseline subtraction and intensity normalization were conducted for all spectra. To identify peak positions and full width at half maximum (FWHM) data, a Lorentz fit was performed for all data. Spectra of the powders after the dispersion treatment were enabled by drying a droplet casted on a silicon wafer in a furnace at $150{ }^{\circ} \mathrm{C}$.

After the production of the nickel matrix composites, the samples were grinded and polished (the finest polish was done using a diamond slurry containing diamond particles of $1 \mu \mathrm{m}$ in diameter) and the distribution of the particles in the matrix was analyzed using a dual beam focused ion beam/field emission scanning electron microscopy (FIB/FE-SEM) workstation (FEI Helios NanoLab 600). The obtained SEM pictures were binarized (a4i analysis software) with a resolution of $62 \mathrm{~nm}$ per pixel to allow for a quantitative distribution analysis of the particle 
agglomerates. Two pixels are needed to define an agglomerate. The mean grain size of the matrix after sintering is measured by EBSD with an EDAX TSL detector attached to the dual beam microscope. The scanned area is of $250 \times 250 \mu \mathrm{m}^{2}$ applying an accelerating voltage of $20 \mathrm{kV}$, a current of $22 \mathrm{nA}$, and a step size of $0.3 \mu \mathrm{m}$. For the analysis, a grain is defined as at least two adjacent points with a maximum misorientation of $5^{\circ}$ beyond which a grain boundary is determined. The acquired raw data was post-processed using confidence index (CI) normalization, followed by the removal of points with CI below 0.1 . The grains intersecting the scan window were excluded from the analysis. Vickers microhardness measurements were performed with a Vickers microindenter (Durascan, Struers) with an indenting force of $0.98 \mathrm{~N}$ (HV 0.1) and a holding time of $10 \mathrm{~s}$. For each sample, 20 measurements at different spots were conducted and averaged.

\section{Results and discussion}

\subsection{Agglomerate size distribution}

The three different CNP materials were first characterized using TEM (Fig. 1). It can be seen that the particles show the characteristic morphology and atomic structure of MWCNT (Fig. 1a and d), OLC (Fig. 1b and e), and ND (Fig. 1c and f) as described in literature. ${ }^{15,16,18,20,21}$ OLCs consist of several spherical shells with a spacing of $0.34-0.36 \mathrm{~nm}$. The primary particles are connected to each other by local sintering and graphitic connections and show a diameter between $5 \mathrm{~nm}$ and $10 \mathrm{~nm} .^{20,21} \mathrm{ND}$ shows a diamond lattice spacing of $0.21 \mathrm{~nm}$ and ND particles are engulfed by amorphous carbon layers. ${ }^{18}$ The average size of ND particles is comparable to that of OLC. The ND and OLC particles exhibit local formation of hard aggregates due to the synthesis process. ${ }^{19,26}$ This feature complicates the dispersion process because of the covalent bonding and sintering of the particles. For CNTs, the high aspect ratio can lead to a mechanical interlocking, resulting in the formation of agglomerates that have to be disentangled. ${ }^{24}$ TEM is a highly

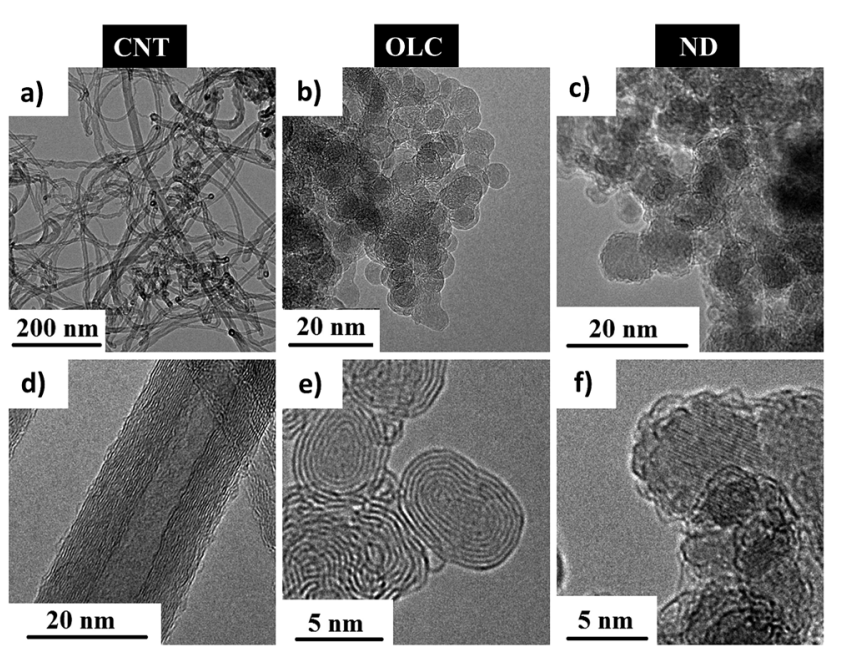

Fig. 1 TEM images of $\operatorname{MWCNT}(a, d), \operatorname{OLC}(b, e)$, and ND (c, f) nanoparticles. localized method to investigate the particle structures or agglomeration sizes in vacuum (i.e., not in the solvent medium). To study the dispersion process of nanoparticles, methods have to be used which can be applied to characterize the particles in liquids, like DLS. As a starting point for the ultrasonic treatment $(t=0)$, the particle powders (CNT, OLC, or ND) were dispersed in ethylene glycol using the homogenizer for $5 \mathrm{~min}$ which breaks-up the initially large agglomerates (up to $1 \mathrm{~mm}$ ). The results are exemplified in Fig. 2, showing the number fraction of particles as a function of the hydrodynamic radius for different treatment times in the ultrasonic bath.

Starting with a hydrodynamic radius of up to $250 \mathrm{~nm}$ after the treatment with the homogenizer $(t=0)$, the hydrodynamic radius of all particles decreases over the first 20 min of ultrasonication. However, longer sonication yields no significant further decrease of the radius, even if the particles are treated for up to $2 \mathrm{~h}$. Therefore, a saturation of the hydrodynamic radius can be found for all kinds of particles at around 40-70 $\mathrm{nm}$. Based on this fact, the dispersions used for the composite manufacturing were all treated for $5 \mathrm{~min}$ in the homogenizer and $20 \mathrm{~min}$ in the ultrasonic bath. This observation is in good agreement with the work of Krüger et al. ${ }^{18}$ who developed a model classifying ND agglomerates in three different sizes, of which only the first two, namely large agglomerates (radius of 10-15 $\mu \mathrm{m}$ ) and intermediate agglomerates (radius of 1-1.5 $\mu \mathrm{m}$ ), could be broken up mechanically in a solvent using an ultrasonic bath. Yet, core aggregates (radius of 50-100 nm) could only be disaggregated by functionalizing the ND. ${ }^{18}$

The agglomerate sized derived from DLS data are complicated by the shape of CNPs. Commonly, DLS is used for analysis only of spherical shaped particles, ${ }^{50}$ the latter being an acceptable approximation for ND and OLC. In contrast to that, the high aspect ratio of a single CNT prohibits the assumption of a spherical form, whereas a CNT agglomerate can be approximated to have an overall spherical shape. In principle, measuring the hydrodynamic radius of CNT agglomerates can be done by DLS. ${ }^{51}$ However, even then, we have to consider that DLS only shows a snap shot of the maximum dispersion grade of the different particles. Therefore, we need to employ a complementary technique to analyze the dispersion stability by means not directly dependent on the shape of the particles. Thus, the next section is dedicated to dispersion stability testing by centrifugal sedimentation analysis.

\subsection{Stability of the dispersions}

Fig. 3 shows the results for the velocity distributions from centrifugal sedimentation analysis of the dispersions treated 5 min in the homogenizer and 20 min of sonication. Both plots present the vol\% of particles sedimenting with a specific velocity. The cumulative plot and the density distribution both show the same data but differ in the way of illustration. The density distribution (Fig. 3b) demonstrates all details of the distribution, for example the mean value, whereas the cumulative version (Fig. 3a) gives the area under the density function. This means the cumulative approach sums up all the volume percentages until a defined velocity. For example for CNT 
(a)

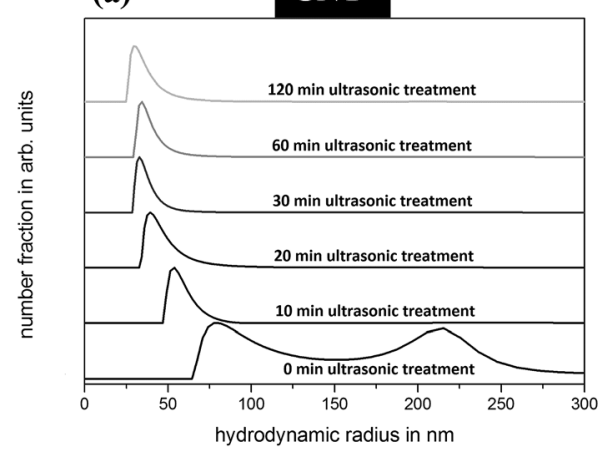

(b)

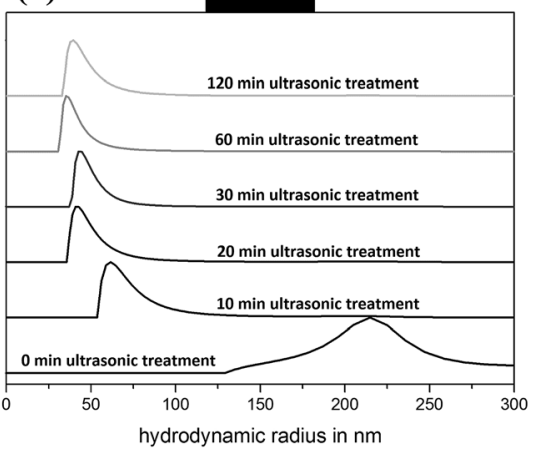

(c) ND

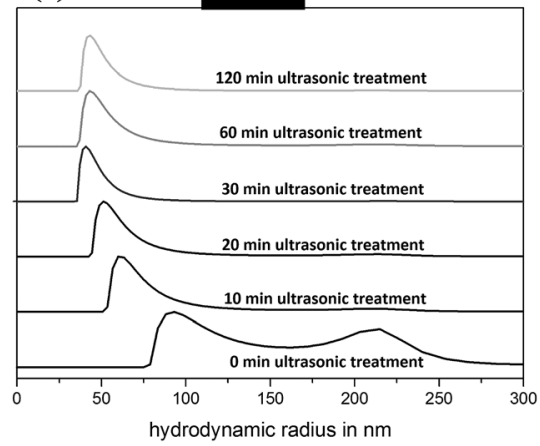

Fig. 2 DLS data of (a) CNT, (b) OLC, and (c) ND in ethylene glycol after different times of ultrasonic treatment. The spectrum of at 0 min is equal to a pre-treatment of $5 \mathrm{~min}$ in the homogenizer to break up the largest agglomerates.

roughly $40 \mathrm{vol} \%$ of the particles sediment with maximum $10 \mu \mathrm{m}$ $\mathrm{s}^{-1}$. This kind of distribution function makes it easier to compare between different datasets because less details are shown compared to the density distribution. Using this
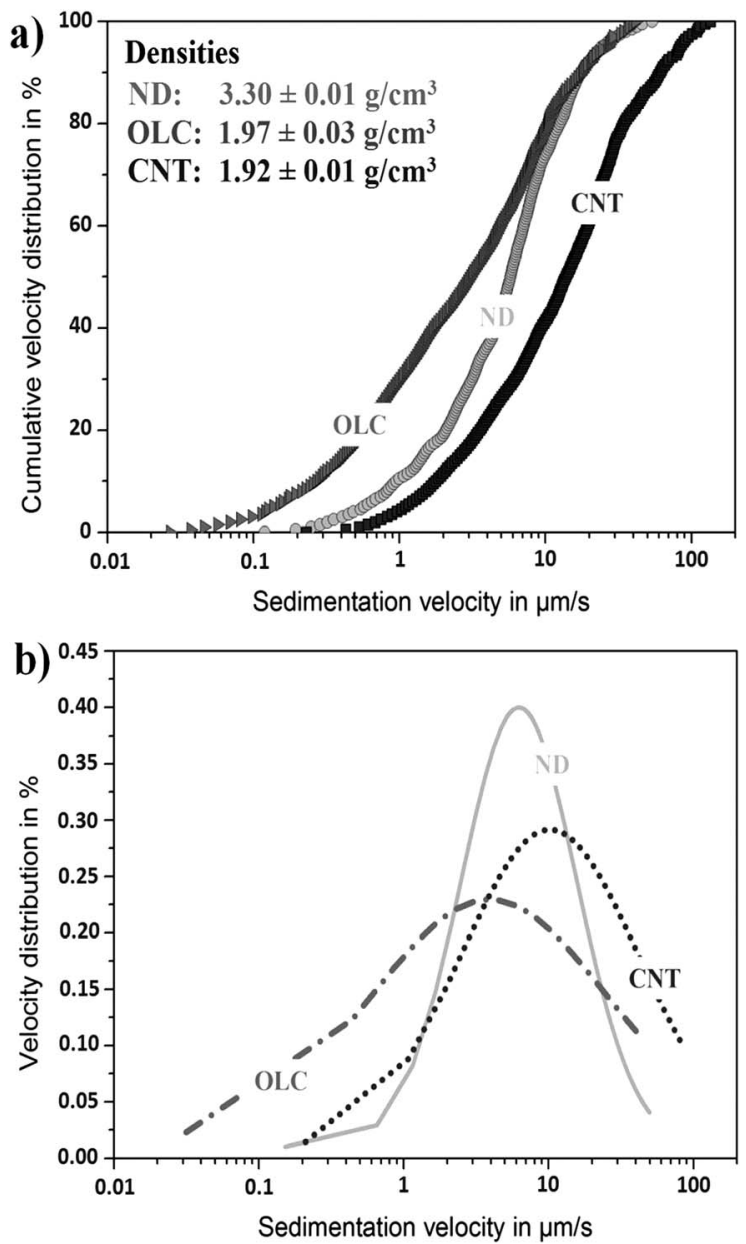

Fig. 3 Sedimentation analysis of CNT, OLC, and ND dispersion in ethylene glycol, showing (a) the sedimentation velocity of the particles as cumulative plot (including particle densities) and (b) the fitted data using a log-normal function. method, the dispersion stability and re-agglomeration behavior of the different particles can be analyzed. The data are strongly dependent on the particle densities. As CNT show the lowest density (similar to OLC) and the highest sedimentation velocity (Fig. 3a), they seem to re-agglomerate most quickly after the dispersion process, thus forming the largest agglomerates. Although the maximum sedimentation velocities of ND and OLC are identical, OLC show a slower sedimentation velocity for up to $\sim 70 \%$ of the distribution than ND (Fig. 3a). However, as ND features a 1.7 times higher density than OLC, the OLC particles seemingly build up larger maximum agglomerates than ND and still sediment slower as their density is much smaller. Consequently, the tendency to re-agglomerate and the dispersion stability differ. The ND dispersion clearly shows the sharpest peak in Fig. 3b, indicating a more homogeneous agglomerate diameter distribution.

In summary, the ND dispersion is the most stable dispersion and most suitable as precursor for a composite manufacturing, whereas the CNT dispersion tends to be the least stable one with the largest agglomerates. Zeta-potential measurements confirm this assumption, allowing for a quantitative evaluation of the dispersion stability. A dispersion showing an absolute value of the zeta-potential higher or lower than $\pm 50 \mathrm{mV}$ is considered to be stable..$^{52}$ The closer the zeta-potential approaches 0 , the more unstable the dispersion becomes. In Table 1, the results of the zeta-potential measurements for the three different particle dispersions types are summarized. The ND dispersions show a slightly higher zeta-potential than CNT and OLC and the zetapotential of OLC appears to be similar to the potential of the CNT dispersions within the scatter of the method. Therefore,

Table 1 Mean values and standard deviations of five zeta-potential measurements for each particle type in ethylene glycol with a concentration of $10^{-3} \mathrm{~mol} \mathrm{dm}{ }^{-3}$ of ammonium acetate

\begin{tabular}{lc} 
Particle & Zeta-potential in $\mathrm{mV}$ \\
\hline CNT & $-21.7 \pm 1.4$ \\
OLC & $-24 \pm 1.9$ \\
ND & $-31.6 \pm 2.4$
\end{tabular}


the dispersion of ND presents itself as the most stable one, which is in good agreement with the results of the sedimentation analysis.

The difference in the sedimentation characteristics between CNT and OLC cannot be explained only by a different zetapotential. As the zeta-potential is similar and the main difference between CNT and OLC is based on their morphology, the different agglomeration behavior can be a consequence of the high aspect ratio of CNT as well as their larger dimension, resulting in a morphologically favored agglomeration effect. ${ }^{24}$ In the case of ND, functional groups may be present on the surface due to the synthesis, resulting in a chemically more stabilized dispersion. ${ }^{19}$

\subsection{Influence of the dispersion process on the structural integrity of the particles}

Conventional processing, for example via ball milling, may yield a good dispersion, but is usually at the expense of structural CNP degradation, leading to a poor reinforcing effect and potential chemical degradation when used in metal matrix composites. $^{29,53}$ In our study, we therefore chose sonication as a gentler method for particle dispersing. To facilitate the deaggregation, a homogenizer is previously used to break up larger agglomerates (up to $c a .1 \mathrm{~mm}$ ), thus reducing the total time needed to disperse the particles in the ultrasonic bath and also reducing the total amount of stress on the particles. However, since the particles are still mechanically highly stressed, a change or variation of the structural state may still be possible and needs to be investigated. ${ }^{51}$

A well-established method to study the defect state of CNPs is Raman spectroscopy. ${ }^{54,55}$ In Fig. 4(a and b), Raman spectra using visible light laser excitation of CNT and OLC can be seen after different ultrasonication treatment times. The first peak at around $1350 \mathrm{~cm}^{-1}$ corresponds to the D-mode, generated by the presence of defects in the CNT or OLC structure, for example a disruption of the aromatic system of $\pi$-electrons in the framework. ${ }^{44,56}$ Close to $1600 \mathrm{~cm}^{-1}$, the G-mode is detected which can be attributed to the tangential vibrations of carbon atoms with $\mathrm{sp}^{2}$-hybridization and can be used to describe their crystalline state. ${ }^{55-57}$ The shoulder at the G-mode at higher wavenumbers can be assigned to the different resonance types of the inner and outermost of the MWCNT. ${ }^{58}$ Finally, the $\mathrm{G}^{\prime}$ mode is observed at around $2700 \mathrm{~cm}^{-1}$, corresponding to an overtone of the defect-related D-mode, and its intensity is proportional to the sample purity level. ${ }^{56}$

A quantitative analysis of the Raman spectra can be accomplished by evaluating the normalized intensity ratios $D / G$ and the $\mathrm{G}^{\prime} / \mathrm{D}$, which are shown as a function of time in the ultrasonic bath for CNT (Table 2) and OLC (Table 3). Since the Raman signal is sensitive to the irradiated volume and in order to obtain a reliable comparison between spectra, it is generally accepted that the correlation should be carried out with intensity ratios. Specifically, the D/G ratio corresponds to the defect density whereas the $\mathrm{G}^{\prime} / \mathrm{D}$ ratio can be correlated with the purity level of graphitic material. ${ }^{55}$ Tuinstra and Koenig ${ }^{59}$ observed that the $\mathrm{D} / \mathrm{G}$ ratio is inversely proportional to the mean domain size in graphitic structures, whereas the domain size is defined as the mean distance between two consecutive defects (e.g., crystal boundary in polycrystalline graphite). Since then, this ratio has been extensively used as an indicator of the defect density in $\mathrm{sp}^{2}$ carbons. DiLeo et al. ${ }^{55}$ showed that the $\mathrm{G}^{\prime} / \mathrm{D}$ ratio is proportional to the purity of a MWCNT sample. The explanation is based on the fact that the $\mathrm{G}^{\prime}$ band is more sensitive than the $\mathrm{D}$ band to carbonaceous impurities, deriving in a very close correlation to average purity values obtained by thermogravimetry. The Raman full width at half maximum (FWHM, $\Gamma$ ) can be correlated with the degree of structural ordering. ${ }^{60}$ Ideally, the defect density should be as low as possible, since defects can act as nucleation centers for failure propagation, leading to reduced mechanical properties. ${ }^{61}$ Based on the data, neither CNT nor OLC show a significant variation of the initial $\mathrm{D} / \mathrm{G}$ or $\mathrm{G}^{\prime} / \mathrm{D}$ ratio as well as the FWHM, even after $2 \mathrm{~h}$ of sonication. This leads to the assumption that both particle types are not significantly altered from the initial structural state during the dispersion process.

The structural integrity analysis of the ND dispersions has to be discussed separately, as the detected bands differ from the ones of CNT and OLC. It is noteworthy that Raman analysis of ND materials required UV-laser radiation in contrast to the use of visible light laser for $\mathrm{sp}^{2}$-hybridized nanocarbons. For all ND
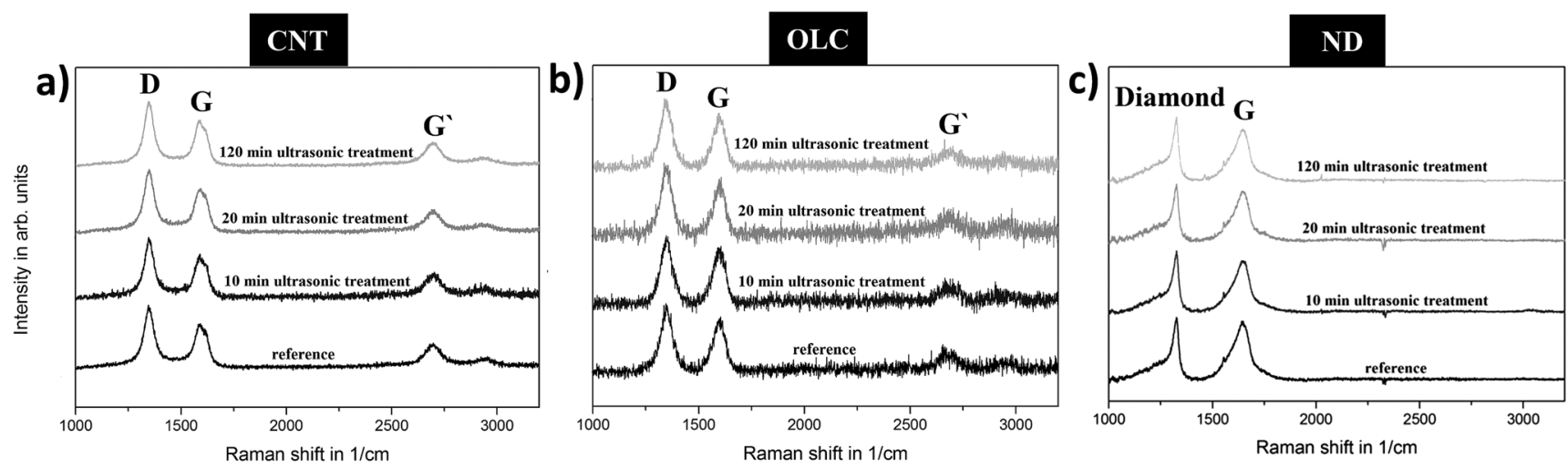

Fig. 4 Raman spectra of CNT (a), OLC (b) and ND (c) on a silicon wafer as a function of the time spent in the ultrasonic bath in the solvent ethylene glycol. 
Table 2 Normalized Raman data of CNT as a function of time spent in the ultrasonic bath. A Lorentz fit was performed to identify the peak positions and the FWHM $(\Gamma)$

\begin{tabular}{llllllll}
\hline $\begin{array}{l}\mathrm{D} \\
\left(1348 \mathrm{~cm}^{-1}\right)\end{array}$ & $\begin{array}{l}\mathrm{G} \\
\left(1593 \mathrm{~cm}^{-1}\right)\end{array}$ & $\begin{array}{l}\mathrm{G}^{\prime} \\
\left(2696 \mathrm{~cm}^{-1}\right)\end{array}$ & $\begin{array}{l}\Gamma_{\mathrm{D}} \\
\text { in } \mathrm{cm}^{-1}\end{array}$ & $\begin{array}{l}\Gamma_{\mathrm{G}} \\
\text { in cm }\end{array}$ & $\begin{array}{l}\Gamma_{\mathrm{G}^{\prime}} \\
\text { in cm }\end{array}$ & $\begin{array}{l}t \\
\text { in min }\end{array}$ \\
\hline 1 & 0.73 & 0.44 & 58.4 & 75.5 & 91.2 & 1.37 & 0.44 \\
1 & 0.75 & 0.49 & 55.8 & 73.3 & 93.5 & 1.33 & 0.49 \\
1 & 0.72 & 0.41 & 59.7 & 75.2 & 91.8 & 1.39 & 0.41 \\
1 & 0.72 & 0.40 & 56.0 & 72.9 & 90.6 & 20 & 1.39 \\
\hline
\end{tabular}

Table 3 Normalized Raman data of OLC as a function of time spent in the ultrasonic bath. A Lorentz fit was performed to identify the peak positions and the FWHM $(\Gamma)$

\begin{tabular}{llllllll}
\hline $\begin{array}{l}\mathrm{D} \\
\left(1348 \mathrm{~cm}^{-1}\right)\end{array}$ & $\begin{array}{l}\mathrm{G} \\
\left(1597 \mathrm{~cm}^{-1}\right)\end{array}$ & $\begin{array}{l}\mathrm{G}^{\prime} \\
\left(2686 \mathrm{~cm}^{-1}\right)\end{array}$ & $\begin{array}{l}\Gamma_{\mathrm{D}} \\
\text { in cm }\end{array}$ & $\begin{array}{l}\Gamma_{\mathrm{G}} \\
\text { in cm }\end{array}$ & $\begin{array}{l}\Gamma_{\mathrm{G}^{\prime}} \\
\text { in cm }\end{array}$ & $\begin{array}{l}t \\
\text { in min }\end{array}$ \\
\hline 1 & 0.81 & 0.40 & 66.2 & 64.5 & 118.3 & 1.23 & 0.40 \\
1 & 0.85 & 0.38 & 64.4 & 63.1 & 127.2 & 1.18 & 0.38 \\
1 & 0.86 & 0.41 & 61.5 & 60.9 & 119.8 & 1.16 & 0.41 \\
1 & 0.79 & 0.32 & 64.7 & 63.0 & 122.5 & 20 & 1.27 \\
\hline
\end{tabular}

Table 4 Normalized Raman data of ND as a function of time spent in the ultrasonic bath. A Lorentz fit was performed to identify the peak positions and the FWHM $(T)$

\begin{tabular}{llllll}
\hline $\begin{array}{l}\text { Diamond } \\
\left(1320 \mathrm{~cm}^{-1}\right)\end{array}$ & $\begin{array}{l}\mathrm{G} \\
\left(1629 \mathrm{~cm}^{-1}\right)\end{array}$ & $\begin{array}{l}\Gamma_{\text {diamond }} \\
\text { in } \mathrm{cm}^{-1}\end{array}$ & $\begin{array}{l}\Gamma_{\mathrm{G}} \\
\text { in } \mathrm{cm}^{-1}\end{array}$ & $\begin{array}{l}\text { Diamond/ } \\
\mathrm{G}\end{array}$ & $\begin{array}{l}t \text { in } \\
\text { min }\end{array}$ \\
\hline 1 & 0.92 & 39.8 & 103.8 & 1.23 & Ref \\
1 & 0.85 & 38.4 & 96.9 & 1.18 & 10 \\
1 & 0.89 & 36.5 & 93.1 & 1.16 & 20 \\
1 & 0.83 & 38.2 & 98.5 & 1.27 & 120 \\
\hline
\end{tabular}

spectra, a down-shifted diamond band at $\sim 1320 \mathrm{~cm}^{-1}$ (diamond) can be observed as well as a band at $\sim 1630 \mathrm{~cm}^{-1}$, often simply tagged as G-mode in analogy to $\mathrm{sp}^{2}$-hybridized (graphitic) carbon (Fig. 4c). However, at least three separate peaks assigned to $\mathrm{O}-\mathrm{H}$ bending vibrations $\left(\sim 1640 \mathrm{~cm}^{-1}\right), \mathrm{C}=\mathrm{O}$ stretching vibrations $\left(1740 \mathrm{~cm}^{-1}\right)$, and finally $\mathrm{sp}^{2}$-hybridized carbon band $\left(1590 \mathrm{~cm}^{-1}\right)$ are superimposing to generate this band. ${ }^{62}$ For the studied ND, we see very little variation of the $I$ diamond and the diamond/G ratio (Table 4). In conclusion, just like for OLC and CNT, we also see no statistically significant variation of the sample's structural ordering after deaggregation.

\subsection{Microstructural investigation of the reinforced nickel matrix composites}

In Fig. 5a-c, SEM pictures (backscattered electrons) of the three different composites are shown all containing $6.5 \mathrm{vol} \%$ of CNPs. The black areas are particle agglomerates, whereas the different shades of gray correspond to different grain orientations of the nickel matrix. Based on the binary pictures in Fig. $5 \mathrm{~d}-\mathrm{f}$ we see that the CNTs yield significantly later aggregates (up to $15 \mu \mathrm{m}$ ) in the nickel matrix composites (Ni CNT) compared to the other composites. The smallest maximum size of agglomerates can be detected for ND nickel matrix composites (Ni ND) and is about 3 $\mu \mathrm{m}$. This is in excellent agreement with the results from sedimentation analysis. Not only the agglomerate sizes differ, but also the distribution homogeneity within the matrix as the small agglomerates in the case of Ni ND are most evenly distributed. The individual agglomerate area of the binary SEM pictures of Fig. $5 \mathrm{~d}-\mathrm{f}$ as a function of the total image area fraction of the agglomerates can be used for a quantitative analysis (Fig. 6). Using the binarized images, black pixels are assigned to agglomerates and white pixels to the metal matrix. The individual pixel size is $62 \mathrm{~nm}$, agglomerates smaller than that cannot be detected, but as the minimum agglomerate diameter measured by DLS is $80 \mathrm{~nm}$, an even higher resolution would not be reasonable. As seen, the agglomerate size distribution is the smallest for Ni ND composites and by far the largest for Ni CNT composites, aligning well with the sedimentation analysis. An evenly distribution of small agglomerates is the most promising reinforcement method for metal matrix composites, since it can homogeneously act on the grain boundaries during grain growth, and thus reduce recrystallization processes enhancing the microstructural thermal stability. ${ }^{63}$ Forces can be homogeneously transferred by the particles as a larger interface between the particles and the matrix is available., ${ }^{2,63}$

Although a large interface area of particles and matrix is required for optimized properties, the formation of a brittle interphase (e.g., carbide formation) at the interface may severely impact the mechanical properties. A brittle interphase could act as nucleation center for failure propagation, as it has the tendency to crack very easily. ${ }^{8}$ To inhibit this, the carbon particles should show a low defect density and a matrix material should be chosen, which does not act as carbide creator. ${ }^{8}$ As it is shown elsewhere, nickel is a reasonable candidate for a comparison of different carbon particles as it only forms 

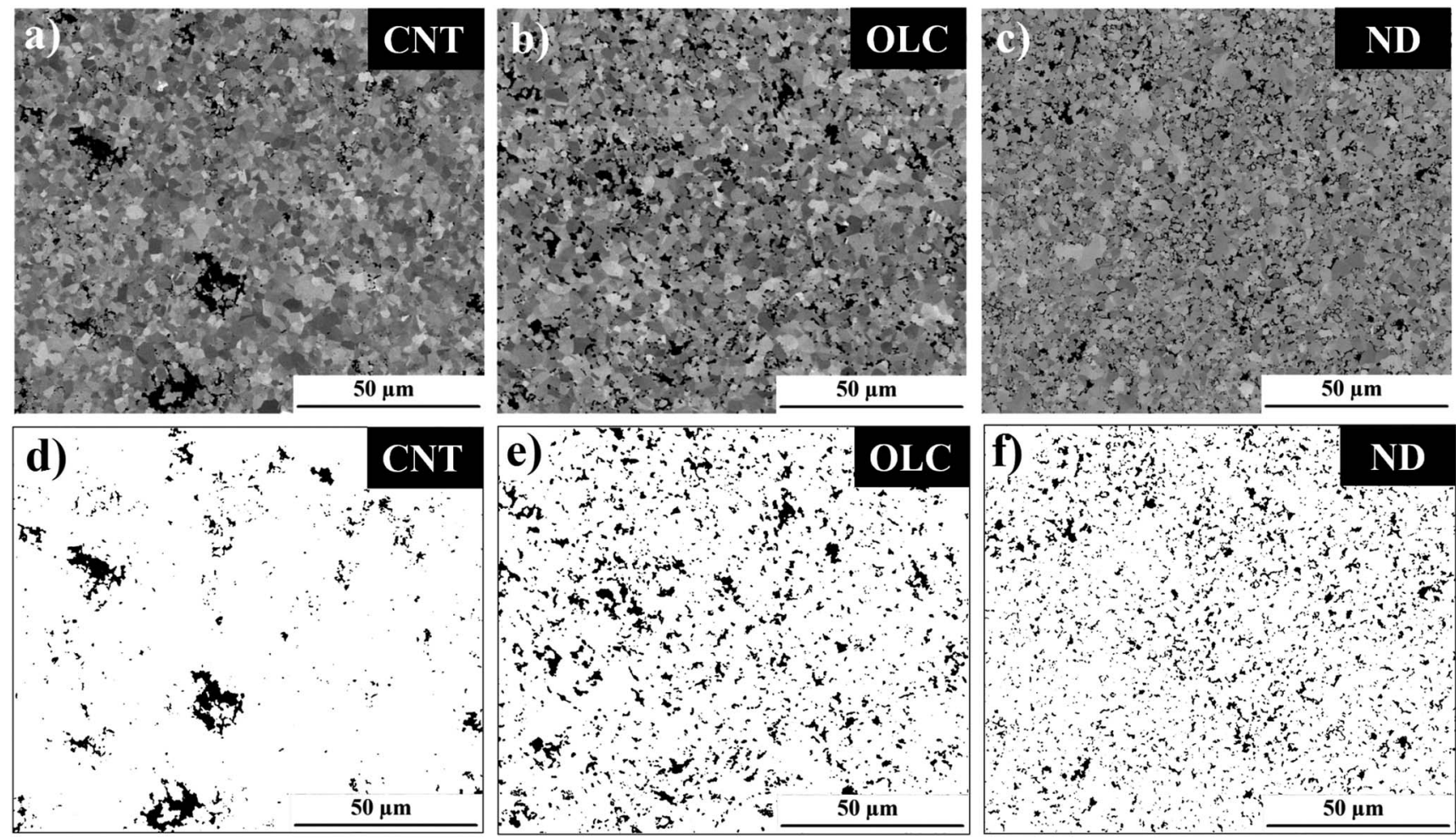

Fig. 5 SEM pictures of (a) CNT-, (b) OLC-, and (c) ND- nickel matrix composites after polishing at the top surface. The uniaxial pressure is applied at the bottom and top surface of the samples. The pictures are binarized for (d) CNT-, (e) OLC-, and (f) ND- nickel matrix composites to enable a better qualitative comparison. The agglomerate sizes decrease significantly and the distribution of the particles becomes more homogenous from (a) to (c) or rather (d) to (f).

metastable carbides; yet, no carbide phases are observed in the case of CNTs for the used conditions in our work. ${ }^{45,64}$ The observed differences in agglomerate sizes and distribution patterns can be well correlated with the results from dispersion analysis. The sedimentation analysis and zeta-potential measurement demonstrated that ND present the most stable dispersion in ethylene glycol. The production of the nickel matrix composites starts with the evaporation of the solvent in

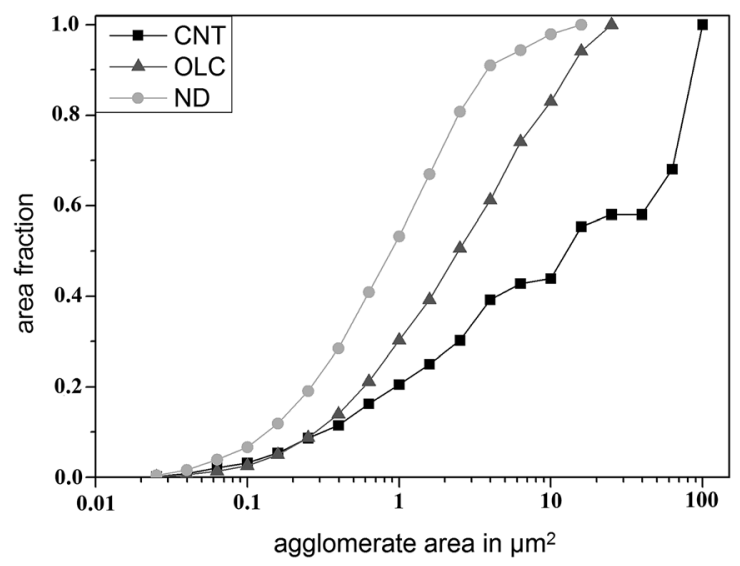

Fig. 6 Individual agglomerate area as a function of the total image agglomerate area (area fraction) in Fig. $5 \mathrm{~d}-\mathrm{f}$ with a resolution of $62 \mathrm{~nm}$ per pixel. a furnace at $150{ }^{\circ} \mathrm{C}$ for $5 \mathrm{~h}$. During this time, the different CNP dispersions re-agglomerate in different ways, which can be explained by the different hybridization states of the carbon atoms and the different particle morphologies. Attractive forces occur between the particles due to the $\pi$-bonding of the $\mathrm{sp}^{2}$ hybridization of carbon atoms in OLC and CNT particles. The large aspect ratio of CNT further leads to possible mechanical interlocking, enabling the emergence of even larger agglomerates than in the case of OLC. Despite the fact that ND show a similar morphology compared to OLC, their $\mathrm{sp}^{3}$-hybridized carbon atoms are subjected to smaller attractive forces between each other as well as a stabilizing effect of the attached functional groups from the synthesis process. This translates into superior dispersion grade and stability than that of both, CNT or OLC dispersions.

The possible grain refinement effect of the different particle types and distributions can be investigated by performing EBSD measurements on an unreinforced nickel reference and on the samples already investigated by SEM (Fig. 7). The mean grain size of the nickel matrix is significantly reduced by the addition of $6.5 \mathrm{vol} \%$ of CNPs up to a factor of 3 for ND and OLC. Furthermore, the Ni CNT composites show larger grains than Ni OLC or Ni ND composites. This can be explained by the agglomerate distribution, as seen from SEM. As the distribution of ND and OLC particles is much more homogenous than the CNT particle distribution, they can homogeneously act as obstacle for the grain boundaries during grain growth through 
a)

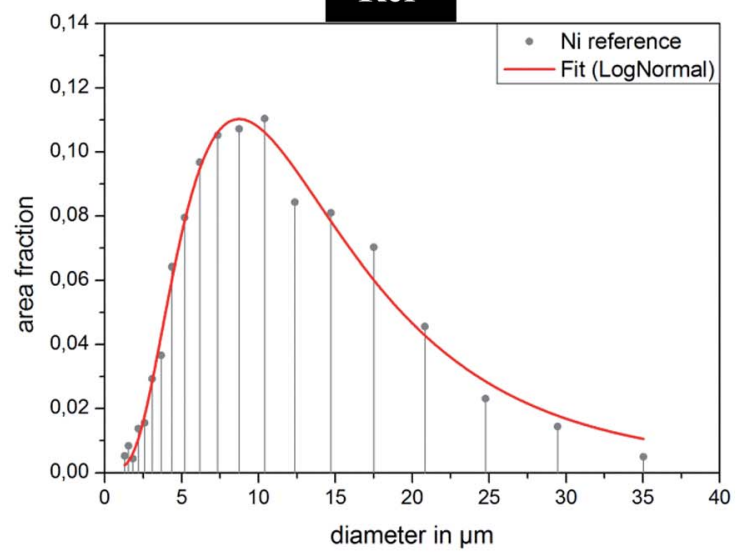

OLC

c)

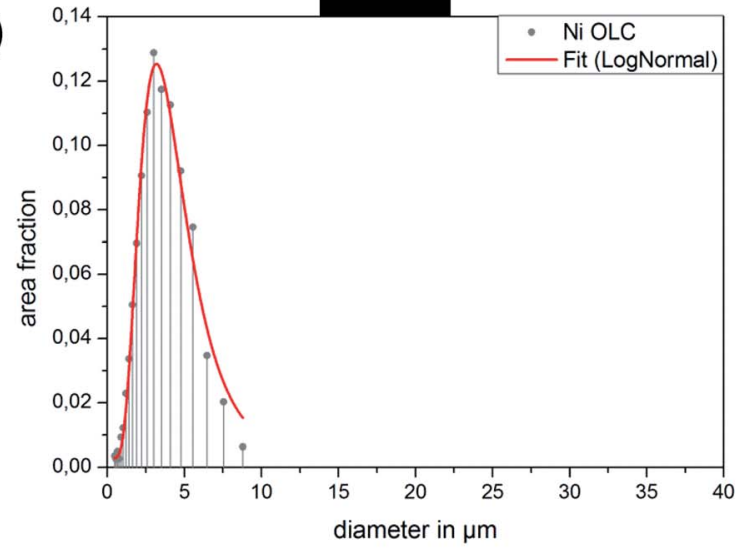

\section{Ref}

b)

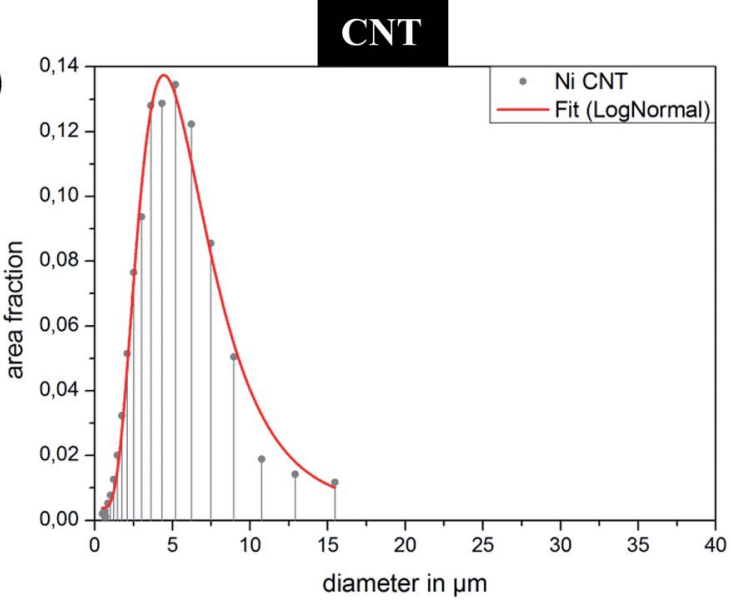

ND

d)

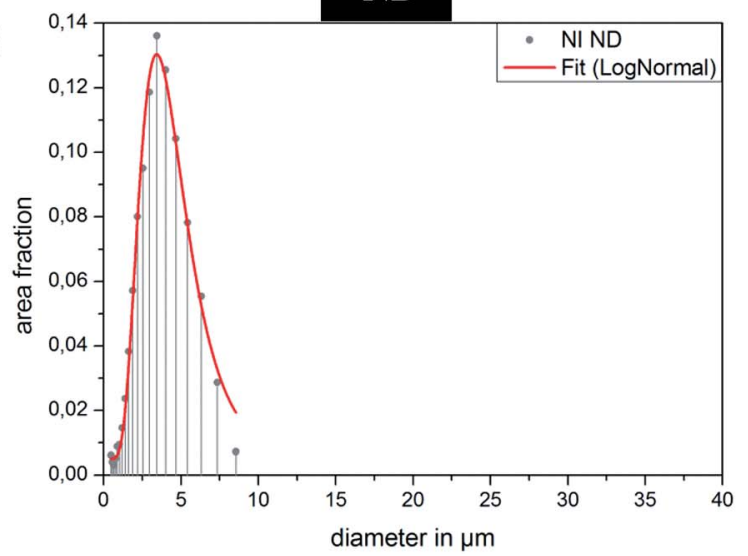

Fig. 7 Grain size distribution of (a) unreinforced nickel reference, (b) Ni CNT, (c) Ni OLC, and (d) Ni ND composites. A LogNormal fit is performed to calculate the mean grain size and the fitted curves are plotted as solid lines (red).

sintering, and thus leading to a finer grain size distribution. ${ }^{45,63}$ Although Fig. 6 shows significant differences in the agglomerate size distribution of the Ni ND and Ni OLC composites, their similar grain size distribution is reasonable. This can be explained as the grain refinement effect is known to be more dependent on the agglomerate distribution within the matrix material rather than on the actual agglomerate size. ${ }^{63}$

Grain refinement directly influences the mechanical properties of the material (e.g., hardness or strength).$^{45}$ We performed Vickers microhardness measurements on the composite samples to analyze this effect. The results can be correlated with the mean grain size, as seen in Table 5 , and the microhardness has been increased when comparing the nickel reference with the CNP reinforced samples. In good correlation to the grain refinement, the Ni CNT sample shows the lowest hardness of the reinforced samples. This can be explained with the larger grain sizes in comparison to Ni OLC and Ni ND, due to the Hall-Petch effect. ${ }^{63,65}$ Although the grain sizes of Ni OLC and Ni ND composites do not differ much, the microhardness of Ni ND is higher than for Ni OLC. This could be due to the smaller ND agglomerate size in Ni ND that allow for a larger interface between $\mathrm{ND}$ and the nickel matrix, being able to efficiently transfer the external forces to a higher amount than the Ni OLC composites. ${ }^{2}$ Rather small agglomerates are known to enable the Orowan mechanism, thus leading to a reinforcing effect of the composite. ${ }^{63}$ The difference in hardness of the composites could also be attributed to differences in the mechanical properties of the OLC and ND particles themselves.

In addition to that, if the particles are distributed unevenly, such as for the Ni CNT composites, depletion zones can exist, that are not reinforced at all. These areas lead to an abnormal grain growth during sintering, ending up with inhomogeneous composite properties. This induces weak spots in the composite

Table 5 Mean grain sizes and mean values of Vickers microhardness measurements of the unreinforced nickel reference and the samples reinforced with CNT, OLC or ND

\begin{tabular}{lcc}
\hline Sample & Mean grain size in $\mu \mathrm{m}$ & HV 0.1 \\
\hline Ni reference & $12.9 \pm 0.5$ & $109 \pm 6$ \\
Ni CNT & $5.7 \pm 0.2$ & $119 \pm 8$ \\
Ni OLC & $4.2 \pm 0.1$ & $125 \pm 4$ \\
Ni ND & $4.0 \pm 0.1$ & $135 \pm 4$
\end{tabular}


material, reducing the mechanical reinforcement effect and generating anisotropy in the properties as can be seen at the higher standard deviation for the hardness of the Ni CNT composites. $^{2,66}$

Summarizing, a low aspect ratio and the $\mathrm{sp}^{3}$-hybridization seems to benefit a small agglomerate size and homogenous distribution of CNPs within a metal matrix composite, thus leading to a strong grain refinement and beneficial mechanical reinforcement effect.

\section{Conclusions}

The dispersion process of non-functionalized MWCNT, OLC, and ND in ethylene glycol using a homogenizer and an ultrasonic bath was studied. The dispersions were used as precursor for the production of nickel matrix composites. In particular, using Raman spectroscopy we found that no damage was inflicted upon the CNPs due to the dispersion process. Yet, although the maximum dispersion grade in the solvent is similar, ND show the best dispersion stability, followed by OLC, and finally MWCNT. This observation is in good agreement with the distribution and agglomerate sizes of the particles in the composite as ND show the smallest and MWCNT the largest agglomerate sizes. In addition, a low aspect ratio and the $\mathrm{sp}^{3}$ hybridization is found to be beneficial for both, a small agglomerate size in the dispersions as well as a homogeneous distribution of small agglomerates within the composite. Finally, a maximum grain refinement by a factor of 3 and a maximum mechanical reinforcement effect of around 25\% (Vickers microhardness) are observed for Ni ND composites. This observation aligns perfectly with the results of the dispersion analysis.

\section{Acknowledgements}

The present work is supported by funding from the Deutsche Forschungsgemeinschaft (DFG, project: MU 959/38-1). L. R., S. S., and F. M. wish to acknowledge the EFRE Funds of the European Commission for support of activities within the AMELab project. This work was supported by the CREATe-Network Project, Horizon 2020 of the European Commission (RISE Project No. 644013). M. Z. and V. P. thank Prof. E. Arzt (INM) for his continuing support. Dr P. Miska is acknowledged for UVRaman measurements at the Institute Jean Lamour (Nancy, France). Dr C. Gachot, Dr A. Rosenkranz, Dr M. Hans, B. Bax, and N. Souza are kindly acknowledged for valuable discussions.

\section{References}

1 J. Y. Hwang, B. K. Lim, J. Tiley, R. Banerjee and S. H. Hong, Carbon, 2013, 57, 282-287.

2 S. R. Bakshi and A. Agarwal, Carbon, 2011, 49, 533-544.

3 D. Nunes, M. Vilarigues, J. B. Correia and P. A. Carvalho, Acta Mater., 2012, 60, 737-747.

4 D. Nunes, J. B. Correia and P. A. Carvalho, Microsc. Microanal., 2013, 19, 2013.
5 V. Livramento, J. B. Correia, N. Shohoji and E. Ōsawa, Diamond Relat. Mater., 2007, 16, 202-204.

6 J. N. Boland and X. S. Li, Materials, 2010, 3, 1390-1419.

7 L. Kumari, T. Zhang, G. Du, W. Li, Q. Wang, A. Datye and K. Wu, Compos. Sci. Technol., 2008, 68, 2178-2183.

8 A. Agarwal, S. Bakshi and D. Lahiri, Carbon NanotubesReinforced Metal Matrix Composites, 2011.

9 D. Nunes, J. B. Correia and P. A. Carvalho, Microsc. Microanal., 2012, 18, 73-74.

10 Y. Li, B. X. Li and W. J. Zou, Appl. Mech. Mater., 2011, 80-81, 683-687.

11 A. Hirata, M. Igarashi and T. Kaito, Tribol. Int., 2004, 37, 899905.

12 K. Miyoshi, K. W. S. Jr, R. L. Vander Wal, R. Andrews and A. Sayir, Tribol. Lett., 2005, 19, 191-201.

13 Y. Gogotsi and V. Presser, Carbon Nanomater., 2014, 279298.

14 S. R. Bakshi, D. Lahiri and A. Agarwal, Int. Mater. Rev., 2010, 55, 41-64.

15 V. N. Mochalin, O. Shenderova, D. Ho and Y. Gogotsi, Nat. Nanotechnol., 2012, 7, 11-23.

16 S. Iijima, Nature, 1991, 354, 56-58.

17 J. Cebik, J. K. McDonough, F. Peerally, R. Medrano, I. Neitzel, Y. Gogotsi and S. Osswald, Nanotechnology, 2013, 24, 1-10.

18 A. Krüger, F. Kataoka, M. Ozawa, T. Fujino, Y. Suzuki, a. E. Aleksenskii, a. Y. Vul' and E. Ōsawa, Carbon, 2005, 43, 1722-1730.

19 M. Zeiger, N. Jäckel, M. Aslan, D. Weingarth and V. Presser, Carbon, 2015, 84, 584-598.

20 H. W. Kroto, J. R. Heath, S. C. O'Brian, R. F. Curl and R. E. Smalley, Nature, 1985, 318, 162-163.

21 D. Ugarte, Nature, 1992, 359, 707-709.

22 K. Bogdanov, A. Fedorov, V. Osipov, T. Enoki, K. Takai, T. Hayashi, V. Ermakov, S. Moshkalev and A. Baranov, Carbon, 2014, 73, 78-86.

23 O. O. Mykhaylyk, Y. M. Solonin, D. N. Batchelder and R. Brydson, J. Appl. Phys., 2005, 97, 1-16.

24 S. D. Bergin, Z. Sun, D. Rickard, P. V Streich, J. P. Hamilton and J. N. Coleman, ACS Nano, 2009, 3, 2340-2350.

25 S. Castro, PhD Thesis, Saarland university, 2007.

26 A. Pentecost, S. Gour, V. Mochalin, I. Knoke and Y. Gogotsi, ACS Appl. Mater. Interfaces, 2010, 2, 3289-3294.

27 N. Pierard, a. Fonseca, J.-F. Colomer, C. Bossuot, J.-M. Benoit, G. van Tendeloo, J.-P. Pirard and J. Nagy, Carbon, 2004, 42, 1691-1697.

28 N. Pierard, A. Fonseca, Z. Konya, I. Willems and G. van Tendeloo, Chem. Phys. Lett., 2001, 335, 1-8.

29 J.-Y. Lee, K.-H. Cho, D.-P. Lim, Y.-B. Lee and D.-S. Lim, Appl. Phys. A, 2007, 88, 693-697.

30 Q. Cheng, S. Debnath, E. Gregan and H. J. Byrne, J. Phys. Chem. C, 2010, 114, 8821-8827.

31 F. Avilés, J. V. Cauich-Rodríguez, L. Moo-Tah, a. May-Pat and R. Vargas-Coronado, Carbon, 2009, 47, 2970-2975.

32 N. Karousis, N. Tagmatarchis and D. Tasis, Chem. Rev., 2010, 110, 5366-5397.

33 C. A. Dyke and J. M. Tour, J. Am. Chem. Soc., 2003, 125, 11561157. 
34 Y.-L. Zhao and J. F. Stoddart, Acc. Chem. Res., 2009, 42, 11611171.

35 L. Vaisman, H. D. Wagner and G. Marom, Adv. Colloid Interface Sci., 2006, 128-130, 37-46.

36 H. Wang, Curr. Opin. Colloid Interface Sci., 2009, 14, 364-371.

37 B. Munkhbayar, M. J. Nine, J. Jeoun, M. Bat-Erdene, H. Chung and H. Jeong, Powder Technol., 2013, 234, 132-140.

38 V. N. Mochalin and Y. Gogotsi, J. Am. Chem. Soc., 2009, 131, 4594-4595.

39 U. Maitra, A. Gomathi and C. N. R. Rao, J. Exp. Nanosci., 2008, 3, 271-278.

40 J. Cheng, J. He, C. Li and Y. Yang, Chem. Mater., 2008, 20, 4224-4230.

41 A. Krueger and T. Boedeker, Diamond Relat. Mater., 2008, 17, 1367-1370.

42 C.-C. Li and C.-L. Huang, Colloids Surf., A, 2010, 353, 52-56.

43 Y. Zhu, X. Xu, B. Wang and Z. Feng, China Particuol., 2004, 2, 132-134.

44 Y. Liu, R. L. Vander Wal and V. N. Khabashesku, Chem. Mater., 2007, 19, 778-786.

45 S. Suarez, F. Lasserre and F. Mücklich, Mater. Sci. Eng., A, 2013, 587, 381-386.

46 G. B. V. Kumar, C. S. P. Rao and N. Selvaraj, J. Miner. Mater. Charact. Eng., 2011, 10, 59-91.

47 M. F. Variava, T. L. Church, A. T. Harris and A. I. Minett, J. Mater. Chem. A, 2013, 1, 8509.

48 S. Suárez, A. Rosenkranz, C. Gachot and F. Mücklich, Carbon, 2013, 1-8.

49 M. D. Chadwick, J. W. Goodwin, E. J. Lawson and P. D. A. Mills, Colloids Surf., A, 2002, 203, 229-236.

50 W. I. Goldburg, Am. J. Phys., 1999, 67, 1152.
51 S. Badaire, P. Poulin, M. Maugey and C. Zakri, Langmuir, 2004, 20, 10367-10370.

52 S. Vallar, D. Houivet, J. El Fallah, D. Kervadec and J. Haussonne, J. Eur. Ceram. Soc., 1999, 19, 1017-1021.

53 H. J. Choi, J. H. Shin and D. H. Bae, Composites, Part A, 2012, 43, 1061-1072.

54 M. A. Pimenta, G. Dresselhaus, M. S. Dresselhaus, L. G. Cançado, A. Jorio and R. Saito, Phys. Chem. Chem. Phys., 2007, 9, 1276-1291.

55 R. A. DiLeo, B. J. Landi and R. P. Raffaelle, J. Appl. Phys., 2007, 101, 064307.

56 J. H. Lehman, M. Terrones, E. Mansfield, K. E. Hurst and V. Meunier, Carbon, 2011, 49, 2581-2602.

57 S. Tomita, T. Sakurai, H. Ohta, M. Fujii and S. Hayashi, J. Chem. Phys., 2001, 114, 7477.

58 X. Zhao, Y. Ando, L.-C. Qin, H. Kataura, Y. Maniwa and R. Saito, Appl. Phys. Lett., 2002, 81, 2550.

59 F. Tuinstra and J. L. Koenig, J. Chem. Phys., 1970, 53, 11261130.

60 S. Suarez, F. Lasserre, O. Prat and F. Mücklich, Phys. Status Solidi, 2014, 211, 1555-1561.

61 K. I. Tserpes and P. Papanikos, Compos. Struct., 2007, 79, 581-589.

62 V. Mochalin, S. Osswald and Y. Gogotsi, Chem. Mater., 2009, 21, 273-279.

63 A. Sanaty-Zadeh, Mater. Sci. Eng., A, 2012, 531, 112-118.

64 J. Y. Hwang, A. R. P. Singh, M. Chaudhari, J. Tiley, Y. Zhu, J. Du and R. Banerjee, J. Phys. Chem. C, 2010, 114, 1042410429.

65 N. Hansen, Scr. Mater., 2004, 51, 801-806.

66 P. Rossi, S. Suarez, F. Soldera and F. Mücklich, Adv. Eng. Mater., 2014, 17, 1017-1021. 